\title{
Sunspot waves and flare energy release
}

\author{
R. Sych ${ }^{1}$, M. Karlický ${ }^{2}$ A. Altyntsev ${ }^{1}$, J. Dudík $^{2,3}$, and L. Kashapova ${ }^{1}$ \\ ${ }^{1}$ Institute of Solar-Terrestrial SB RAS, Lermontov st. 126a, 6640333 Irkutsk, Russia \\ e-mail: sych@iszf.irk.ru \\ 2 Astronomical Institute of the Academy of Sciences of the Czech Republic, 25165 Ondřejov, Czech Republic \\ ${ }^{3}$ RS Newton International Fellow, DAMTP, CMS, University of Cambridge, Wilberforce Road, Cambridge CB3 0WA, UK
}

Received 19 August 2014 / Accepted 25 February 2015

\begin{abstract}
Context. We study the possibility of flare process triggering by waves propagating from the sunspot along a magnetic loop (channel) to a nearby flare site.

Aims. We present a relationship between the dynamics of $\sim 3$-min slow magnetoacoustic waves in the sunspot and flare emergence process. Waves propagating in the magnetic channel whose one foot is anchored in the umbra represent the disturbing agent responsible for triggering the flare energy release.

Methods. We applied time-distance plots and pixel wavelet filtration methods to obtain spatio-temporal distribution of wave power variations in radio and SDO/AIA data. To find the magnetic channel, we used potential magnetic field extrapolation of SDO/HMI magnetograms. The propagation velocity of wave fronts was measured from wave locations at specific times.

Results. In the correlation curves of the $17 \mathrm{GHz}(\mathrm{NoRH})$ radio emission, we found a monotonous energy amplification of the 3-min waves in the sunspot umbra before the 2012 June 7 flare. This amplification was associated with an increase in the length of the oscillatory wakes in coronal loops (SDO/AIA, $171 \AA$ ) prior to the flare onset. A peculiarity of the flare is the constant level of the flare emission in soft X-rays (RHESSI, 3-25 keV) for $\sim 10 \mathrm{~min}$ after the short impulsive phase, which indicates continuing energy release. Throughout this time, we found transverse oscillations of the flare loop with a $30 \mathrm{~s}$ period in the radio-frequency range (NoRH, $17 \mathrm{GHz}$ ). This period appears to be related to the 3-min waves from the sunspot. The magnetic field extrapolation based on SDO/HMI magnetograms shows the existence of the magnetic channel (waveguide) connecting the sunspot with the energy release region.

Conclusions. We analysed the sunspot 3-min wave dynamics and found a correlation between the oscillation power amplification and flare triggering in the region connected to the sunspot through the magnetic channel. We propose that this amplified wave flux triggered the flare. The flare occurred because of this amplified flux, however, and because a sufficient amount of free magnetic energy was accumulated in close vicinity to the magnetic channel prior to the flare. Furthermore, because of loop heating, the wave velocity (sound velocity) increased with the penetration of waves into the energy release site. The heating is shown to be able to proceed after the flare main peak owing to a further energy pumping in the form of waves from the sunspot and additional reconnection episodes in the flare region.
\end{abstract}

Key words. Sun: flares - Sun: oscillations - Sun: X-rays, gamma rays

\section{Introduction}

The initialization of solar flares remains an unsolved problem. Early ideas on how the initialization might occur were described by Norman \& Smith (1978). They argued that flare process cannot start in the entire flare volume at one instant, and proposed that the flare onset was localized in a small part of an active region, from which the energy release extends as dissipation spreading process throughout the flare volume. Two types of agents that may lead to this kind of a dissipation process were addressed: electron beams and shock waves. These agents can trigger flares at large distances from their initial locations, causing sympathetic (simultaneous) flares or leading to a sequential flare energy release in one active region (Liu et al. 2009; Zuccarello et al. 2009). These triggering processes were numerically studied by Karlický \& Jungwirth (1989) and Odstrčil \& Karlický (1997). Karlický \& Jungwirth (1989) assumed that electron beams, penetrating into the current sheet in the magnetic reconnection region, generate Langmuir waves. Then, using the particle-in-cell model, the authors studied the effects of these electrostatic waves on the plasma system. Sufficiently strong Langmuir waves were found to be able to generate ion-sound waves through the three-wave decay process (Bárta \& Karlický 2000). These ion-sound waves increase electrical resistivity in the current sheet system, which results in the onset of the energy dissipation. Thus, the electron beams are able to cause magnetic reconnection. Odstrčil \& Karlický (1997) studied the mechanism for the flare trigger by shock waves. They used a 2D magnetohydrodynamic model with the MHD shock wave propagating towards the current sheet. A portion of the shock wave passed through the sheet, and the rest was reflected. Nothing occurred at the very beginning of the wave-current sheet interaction. However, after some time, specific plasma flows around the current sheet were formed, which led to the start of magnetic reconnection. This shows that for reconnection to be triggered, the enhanced electrical resistivity as well as the plasma flows are important.

Furthermore, spatio-temporal dynamics of magnetic loops and flux-ropes may also lead to the onset of the magnetic reconnection (e.g. Aulanier et al. 2012). Using the SDO/AIA data, Dudík et al. (2014) presented a motion of magnetic loops with the slipping reconnection before the impulsive phase of the 2012 July 12 eruptive flare. At this flare phase, the footpoints exhibited significant motions at speeds of about several tens of kilometers 
per second along expanding flare ribbons. Further, after the peak of the energy release, loop motions and reconnections continued. At this time, a weak coronal mass ejection visible in the SDO/AIA 131 A wavelength was also observed.

Besides the already mentioned beams and shocks, magnetohydrodynamic waves can also lead to triggering reconnection. Nakariakov et al. (2006) showed that these waves are strongly amplified near an X-point and thus trigger reconnection. Sych et al. (2009) reported an increase of a power maximum of slow magnetoacoustic waves propagating from sunspots into the flare region well before $(\approx 20 \mathrm{~min})$ the onset of the observed flare. It was proposed that these waves, propagating along magnetic loop channels, penetrate into the current sheet region and initiate the flare process.

The goal of this paper is to study the temporal and spatial dynamics of 3-min sunspot oscillations in the active region NOAA 11494 on 2012 June 7, and their relation to the start and evolution of a small C-class flare that originated next to the sunspot. This flare was thoroughly studied by Kotrč et al. (2013). We investigated the dynamics of the flare loop in the radio, ultraviolet, and X-ray ranges, and the related sources of umbra oscillations. We used the time-distance plot method to detect wave processes along the selected directions. To calculate narrow-band images of the sources in the selected spectral range, we applied the pixel wavelet filtration (PWF-analysis; Sych \& Nakariakov 2008) and the fast Fourier transform (FFT-analysis). Magnetic field extrapolation was performed using the Fourier transform method of Alissandrakis (1981) and Gary (1989).

The paper is organized as follows: in Sect. 2, we describe our observational data and processing methods. In Sect. 3, we present the data analysis and results, and in Sect. 4, a scenario of the flare is shown. Section 5 provides conclusions.

\section{Observations}

The 2012 June 7 flare occurred in the active region NOAA 11494 at 05:00-06:10 UT with the maximum near 05:56 UT. The flare importance was $\mathrm{C} 1.5$. The active region was located near the central meridian (S18, W20) and involved a large symmetric sunspot of N-polarity. The size of the umbra was $\sim 12$ arcsec, the penumbra $\sim 24$ arcsec. We use the images obtained in the radio (17 GHz, R-L) as well as in extreme ultraviolet (EUV) $94 \AA$ and $171 \AA$ ranges, together with the correlation curves (17 GHz, R-L), dynamic radio spectra from Callisto BLEN 200$800 \mathrm{MHz}$ ) and Ondřejov Observatory (800-2000 MHz), and the soft X-ray data (6-12 keV).

\subsection{Radio data}

The temporal dynamics of the flare radio source was analysed by processing the three-dimensional $(2 \mathrm{D}$ images $\times$ time) cube images of Nobeyama Radioheliograph (NoRH) at $17 \mathrm{GHz}$. A size of the region of interest (ROI) with a symmetrical sunspot in the centre is $120 \times 120^{\prime \prime}$. The radio images of the flare region were synthesized every $10 \mathrm{~s}$ during 05:00-06:30 UT. We used the Fujiki method for cleaning "dirty" radio maps. This technique was designed specially for a study of flare sources. The size of the synthesized diagram was about $12^{\prime \prime}$. To remove the differential rotation and shear motion of sources, we used the IDL program drot_map.pro from the Solar Soft library. This allowed us to align the radio images with respect to the initial time at 05:00 UT in the heliocentric coordinate system.
We prepared a 3D cube of spatial dynamics of the sources in time and superimposed individual radio frames on EUV images.

Data in intensity and polarization channels at $17 \mathrm{GHz}$ give the similar results for the radio sources associated with unipolar sunspots. However here we only present an analysis of the polarization channel data, because they correspond better to the nature of the cyclotron mechanism of the radio emission. The polarized emission at $17 \mathrm{GHz}$ is generated at the third harmonic of the gyrofrequency in the regions with strong magnetic fields $\sim 2-3 \mathrm{kG}$ (Shibasaki et al. 1994; Nindos et al. 2000). These conditions are fulfilled in sunspots in which the field strength in the umbra reaches these values. Considering a position of the 3-min oscillation sources in the sunspot umbra (Sych \& Nakariakov 2014; Yuan et al. 2014; Reznikova et al. 2012), we chose the polarization channel. In this case, the polarized radio source mainly coincides with the umbra, where the maximum variation of the radio brightness is observed.

For a quick view of oscillation activity in different times, we used the correlation curves (Shibasaki 2001). The crosscorrelation signals from the pair of antennas are the complex Fourier components (Thompson et al. 1986). We used the average values of the visibility amplitude obtained from long baseline antennae. The resulting correlation signals is very sensitive to changes in the compact sources that are smaller than $24^{\prime \prime}$. This approach allows for the detection of non-stationary processes occurring in sunspots, flare kernels, etc. We used the circular polarization channel for analysis of the radio images. During our observations, only one strongly polarized radio source of the R-polarity, associated with a sunspot, was located on the solar disk. All bursts and oscillations on correlation curves were associated with this source. Correlation coefficients were obtained for three hours (03:30-06:30 UT) of observation with a cadence of $1 \mathrm{~s}$. This allows us to obtain a good spectral resolution of the wavelet spectrum and to track the frequency and temporal dynamics of the 3-min harmonic before, during, and after the peak of the flare energy release. Unfortunately, the end of NoRH observations at 06:30 UT prevents us from studying the post-flare phase. A number of radio oscillations with the 3 -min period before the flare was $\sim 48$ (12 trains), and at the decline stage $\sim 12$ (3 trains) were detected. This is sufficient to obtain a reliable information about relationship between sunspot oscillations and flare activity.

\subsection{Data in EUV}

We used the time-series of images in EUV obtained in the $171 \AA$ and $94 \AA$ bands with the Atmospheric Imaging Assembly instrument (AIA, Lemen et al. 2012) onboard the Solar Dynamics Observatory (SDO/AIA, Pesnell et al. 2012). We chose observations in two AIA passbands only, as these sufficiently show both the hot flare plasma (AIA $94 \AA$, dominated by Fe XVIII under flare conditions e.g. Petkaki et al. 2012), as well as cooler active region emission together with the transition-region emission from the flare ribbons, and the cooling flare loops (AIA $171 \AA$, Fe IX). The other AIA channels in general show morphology similar to either AIA $94 \AA$ or $171 \AA$ because of the nature of their thermal responses (e.g. Del Zanna 2013).

The temporal resolution was $12 \mathrm{~s}$ and the spatial resolution between pixels was 0.6 arcsec. The duration of the observation used here was $1.5 \mathrm{~h}$ (05:00-06:30 UT), which allowed us to study the sunspot oscillations within $0.5-30$ min periods, and to trace the evolution of the flare process in the active region. 

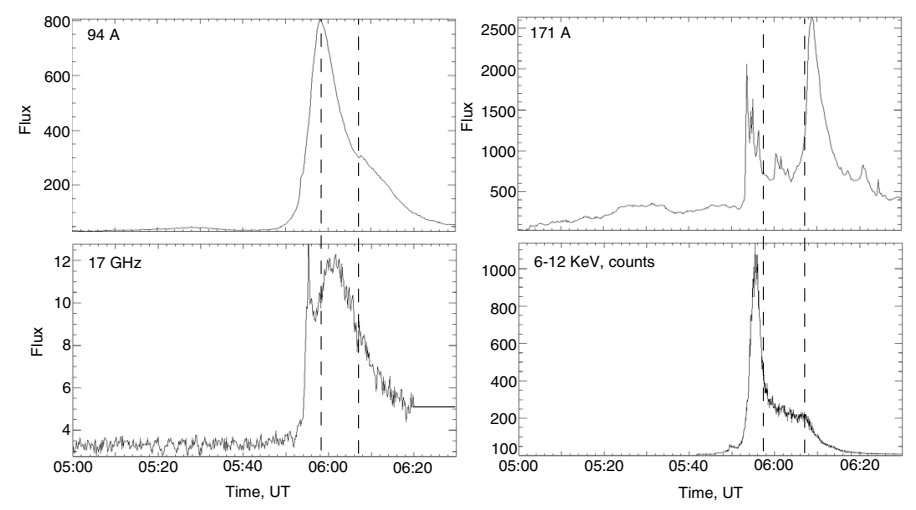

Fig. 1. Light curves of the 2012 June 7 flare in EUV channels (SDO/AIA, $94 \AA$ and $171 \AA$ ), radio (NoRH, $17 \mathrm{GHz}$ ) and X-ray (RHESI, 6-12 keV). The flat stage is shown by vertical dashed lines 05:58-06:08 UT.

Level 1 AIA images were obtained from the online repository ${ }^{1}$. The comparison between different wavelengths is facilitated by converting the data to a Level 1.5 with additional data transformation, such as moving, rotating, and scaling images. For this, we used the IDL program aia_prep.pro from the Solar Soft package. The source selection was performed manually, by assigning the active region center coordinates and the site dimensionality. The differential rotation of the studied object within the observation day was removed by introducing an integer shift using the algorithm implemented at the website. This allowed us to align images in different wavelengths, overlay them, and investigate their dynamics.

\section{Data analysis and results}

\subsection{Flare profiles}

Figure 1 presents the flare flux profiles obtained in the radio (NoRH, $17 \mathrm{GHz}$, intensity), in ultraviolet (SDO/AIA $171 \AA$ and $94 \AA$ ), and in the soft X-ray (RHESSI) ranges. One can see that the profiles have three peaks at different times. The first peak at $\sim 05: 55$ UT is observed in most of the wavelengths with different intensities. The second peak near 06:00 UT has the maximum at the chromospheric level (radio, $17 \mathrm{GHz}$ ) diminishing towards the corona (171 $\AA$ ). The peak in the $94 \AA$ channel shows the existence of high-temperature processes. The third peak at $\sim 06: 09$ UT is only visible in the $171 \AA$ channel line. This is evidenced by a spatial-height dynamics of the flare energy release sources. Note the formation of a flat interval on the X-ray profile at 05:58-06:06 UT. The flare loops are spatially near the sunspot.

\subsection{Radio data analysis}

\subsubsection{Dynamic spectra analysis}

The Callisto BLEN (200-800 MHz) (Fig. 2) and Ondřejov (800$2000 \mathrm{MHz}$ ) radio spectra show that the radio flare starts with type-III bursts at 05:53:40-05:54:24 UT in the 200-400 MHz range. It shows that these processes started at about of $400 \mathrm{MHz}$, i.e. at the plasma density $n_{\mathrm{e}}=1.98 \times 10^{9} \mathrm{~cm}^{-3}$ (fundamental emission) or $n_{\mathrm{e}}=4.98 \times 10^{8} \mathrm{~cm}^{-3}$ (harmonic emission). Then at 05:54:24-05:55 UT broadband (200-2000 MHz) type-III bursts together with the reverse drift bursts in the $800-1300 \mathrm{MHz}$ appeared. These bursts, which designated the impulsive phase of

\footnotetext{
1 http://www.lmsal.com/get_aia_data/
}

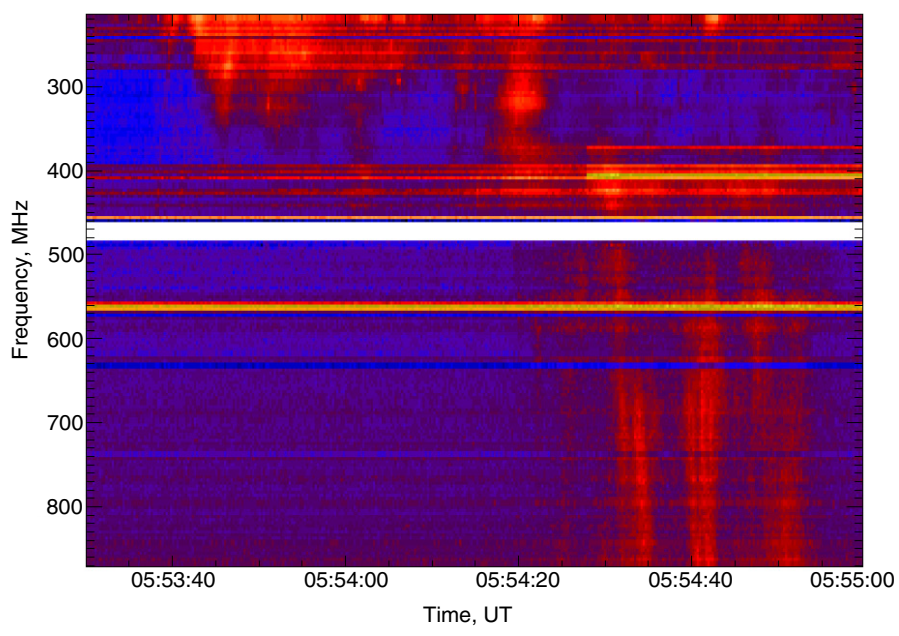

Fig. 2. Callisto BLEN radio spectrum observed during the 2012 June 7 flare.

the flare, are followed by a noise storm in the $200-300 \mathrm{MHz}$ range.

\subsubsection{Dynamics of oscillations before the flare}

To study the sunspot oscillations and their relationship with the flare process, we analysed the behaviour of the correlation coefficients obtained by Nobeyama radioheliograph at $17 \mathrm{GHz}$ in circular polarization channel (Fig. 3a). We observed moderate activity with some radio bursts and high-frequency oscillations during the whole daytime observation. Horizontal lines show (1) the interval before the flare (burst) at 03:30-05:55 UT, and (2) in the postflare stage at 05:55-06:30 UT. Figure 3b demonstrates that the correlation coefficients have significant temporal variations near the 3-min period. There are low-frequency wave trains with periods near $\sim 12-20 \mathrm{~min}$, which is in good agreement with previously published results (Sych et al. 2009; Abramov-Maximov et al. 2011). We performed spectral processing of the signal correlation curves (wavelet transform) during 03:30-06:30 UT. The wavelet spectrum shows (Fig. 3d) a significant increase in the power of the 3-min oscillation trains before the flare peak. We find the same behaviour for the oscillation amplitude (Fig. 3b). Oscillations start to grow two hours before the flare. We observed the maximum power of the 3-min oscillation train at $\sim 05: 30$ UT i.e. about 20-30 min before the main peak of the flare energy release. Immediately after the flare peak, we observed a sharp decrease of the oscillations (interval 2 in Fig. 3), lasting $\sim 20 \mathrm{~min}$. Then, until the end of observations NoRH at 06:30 UT, the oscillations started to rise to their average level observed before the flare at 03:30-03:50 UT. During each train, we observed multidirectional drifts of period in time range from 2 to $4 \mathrm{~min}$ (Fig. 3d). There is a decrease of the drift variation over the period range, as well as the growth of the maximum oscillation period before the flare.

\subsubsection{Spatial evolution of the flare radio source}

To obtain the radio source spatial pattern prior to and during the flare, we synthesized the radio sources (NoRH, $17 \mathrm{GHz}$ ) over 05:00-06:20 UT with the $10 \mathrm{~s}$ temporal resolution. Figure 4 shows individual images in the intensity channel (black continuous contours) and circular polarization (white color, dotted contours), superposed on coronal images in ultraviolet (SDO/AIA, 

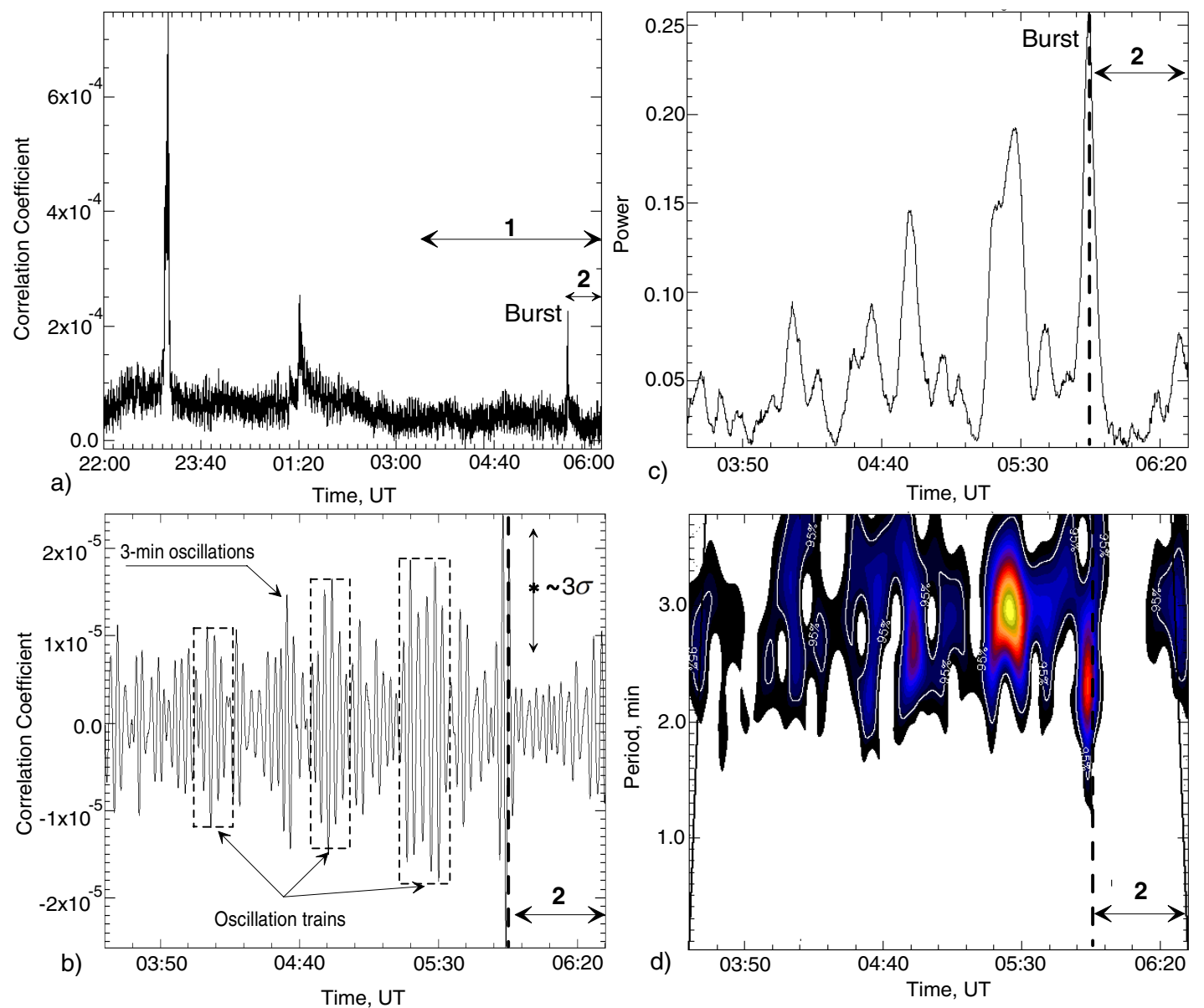

Fig. 3. a) Correlation curve for the polarization channel (NoRH, $17 \mathrm{GHz})$ during daytime observations. The horizontal lines shows the interval before the flare (burst) at 03:30-05:55 UT (1) and at the post-flare stage, at 05:55-06:30 UT (2). b) 3-min variation of correlation coefficients with removed low-frequency trend. The $3 \sigma$ error bar is added. c) Power variation of correlation coefficients centred at 3-min period. d) Wavelet power spectrum of oscillations. The flare (burst) maximum is shown by vertical broken line.

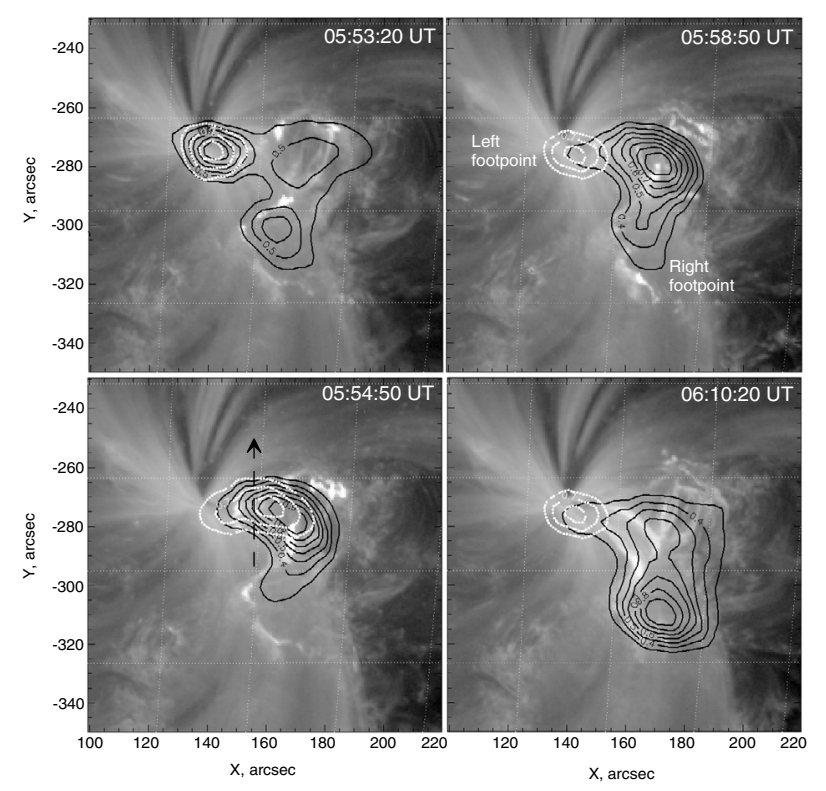

Fig. 4. Temporal evolution of the microwave burst (NoRH, $17 \mathrm{GHz}$ ) observed during the 2012 June 7 flare. The black solid lines and the white dotted lines corresponds to the intensity in maximum levels and polarization in levels of minimum intensity, respectively. The background is an AIA $171 \AA$ image. The arrow shows the path of scans for the timedistance plot.
$171 \AA)$. We obtained the images for times prior to the flare (05:53:20 UT) and for energy release peak times (05:54:50 UT, 05:58:50 UT, and 06:10:20 UT). A loop-like source in the intensity channel prior to the flare at 05:53:20 UT is evident. One of its footpoints is anchored in the umbra. Contours of the radio loop approximately coincide with the coronal loops visible in the EUV $171 \AA$ channel. A displacement of the loop top (in projection) and the formation of a new flare loop occur at the time of the first flare maximum at 05:54:50 UT. White dotted contours indicate the strongly polarized sunspot. This polarized source (generated by the gyro-synchrotron emission) starts to form along the loop left foot in the umbra, indicating heating a part of the loop anchored in the sunspot. The heating is related to the 3-min wave trains and with their growth (Fig. 3). During the period corresponding to the second and third flare maximum, the radio loop flattens (Fig. 4) coinciding with the new coronal loops originated in ultraviolet emission at $171 \AA$.

\subsubsection{Radio loop oscillations}

To study the sunspot wave dynamics and the flare loop oscillations during the flare, we used the time-distance plot constructed from the radio images (NoRH, $17 \mathrm{GHz}$ ) in the polarization channel. We averaged over two pixels $\left(\approx 4.8^{\prime \prime}\right)$ across the scanning direction shown by the arrow in Fig. 4 at 05:54:50 UT. We used global wavelet spectra to obtain the spectral components. 

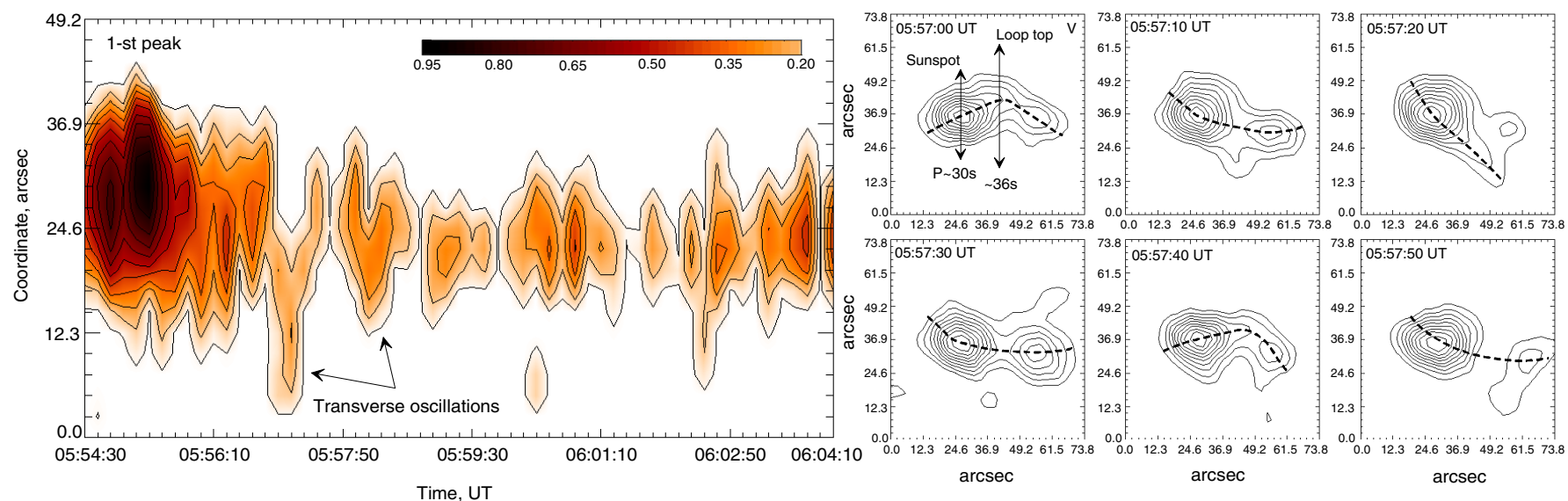

Fig. 5. Induced transverse oscillations of flare loop in $17 \mathrm{GHz}(\mathrm{NoRH})$ polarization channel. Left panel: time distance plots of oscillations in the loop top. Right panel: images of radio sources during transverse oscillations at 05:57:00-05:57:50 UT.

Figure 5 shows that during the flare, well-defined transverse loop oscillations emerge with a mean period of $\approx 35 \mathrm{~s}$. These oscillations were noted earlier in a number of papers (e.g. Kupriyanova et al. 2013). Prior to the present flare, no periodicity was observed out of the sunspot. Then, the V-shaped forms were generated as a result of recurrent motions (Fig. 5, left panel). The maximum spatial shift of the oscillating loop was observed during the flare peak at 05:54:30-05:56:20 UT with gradual damping during the flat X-ray stage (Fig. 1).

A periodic transverse component of the oscillations was found in the whole loop. However, in the umbra at the footpoint of flare loop, the spatial shifts due to oscillations are minimal. The oscillation power grows towards the middle of the loop foot. The spatial direction of periodical displacements from minimal values ( $\sim 30 \mathrm{~s}$ ) in the loop footpoint to maximal in the loop top $(\sim 36 \mathrm{~s})$ is shown by arrows (Fig. 5, right panel). Also, the wellknown 3-min sunspot longitudinal oscillations with $\sim 185-223 \mathrm{~s}$ periods are observed.

\subsection{Analysis of EUV data}

The radio analysis of the transition-region emission, presented above, shows the existence of an energy boost in propagating waves before the flare onset in a sunspot. A uniform waveguide appears in the form of a new loop-like radio source along which the waves start to propagate towards the flare region. To study these processes in the corona, we analysed the SDO/AIA observations in the $171 \AA$ and $94 \AA$. Accounting for a significant difference between the NoRH angular resolution $(\sim 10 \mathrm{arcsec})$ and SDO $(\sim 0.6 \operatorname{arcsec})$, a more detailed picture of these processes can be expected.

\subsubsection{Spatial evolution of flare loops}

First brightenings were recorded at 05:49:44 UT. Figure 6 shows the evolution of flare loops prior and during the flare (05:51:36, 05:54:24, and 06:10:36 UT). The flare started with a brightening of the hot $94 \AA$ loop structure whose footpoints were anchored in its colder $171 \AA$ A ribbons. Simultaneously, the distance of these ribbons increased. These changes occurred during the first maximum of the flare profiles (Fig. 1) and they corresponded to those in the radio (Fig. 4). One of the loop footpoints is anchored in the sunspot. The second flare peak at $\sim 06: 00$ UT was associated with the loop brightness maximum in the $94 \AA$ hot coronal line
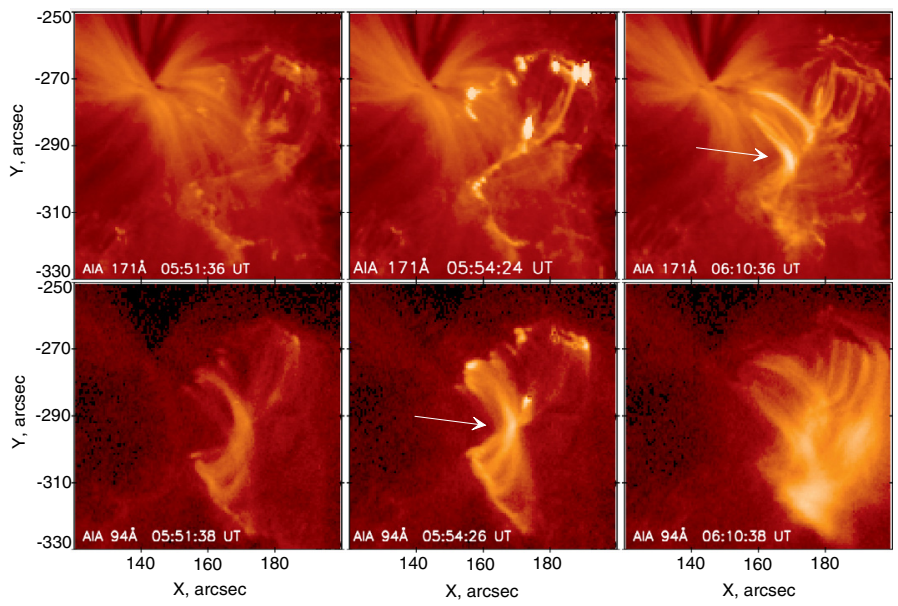

Fig. 6. Flare loop evolution in the $171 \AA$ (upper row) and $94 \AA$ (bottom row) lines during the 2012 June 7 flare at three times: 05:51:36 (:38), 05:54:24 (:26), and 06:10:36 (:38) UT. The arrows show the location of changing magnetic field structure (from sheared to a near-potential one), see the plots at 05:54:26 and 06:10:36 UT.
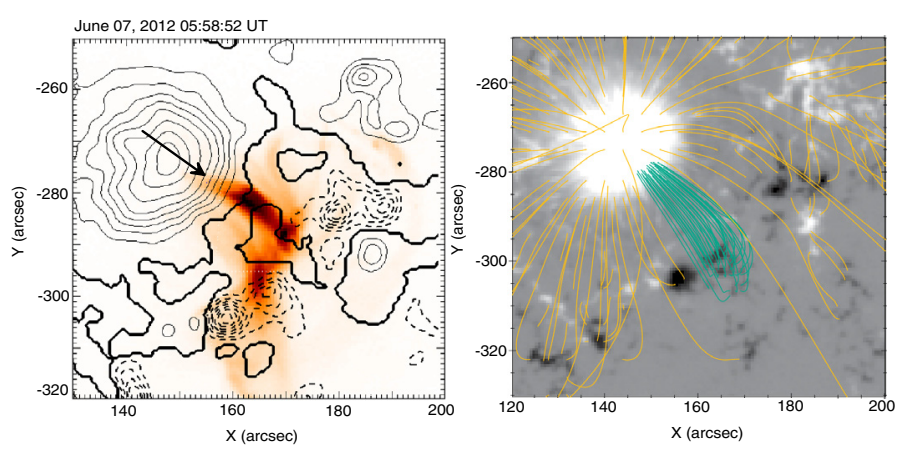

Fig. 7. Left panel: image of flare loops in hot temperature channel (94 $\AA$ ) at 05:58:52 UT. Contours show the magnetic field at the photosphere (SDO/HMI magnetogram). The arrow shows the scanning direction used in construction of the time-distance plot in Fig. 8. Right panel: extrapolated magnetic field lines. The green lines mean the magnetic channel (waveguide) for 3-min oscillations.

(Fig. 7). Its onset coincided with the start of the 05:58-06:06 UT flat stage on the soft X-ray 6-12 keV (RHESSI) time profile. The flare loop visibility maximum in the $171 \AA \AA$ cold coronal line falls 

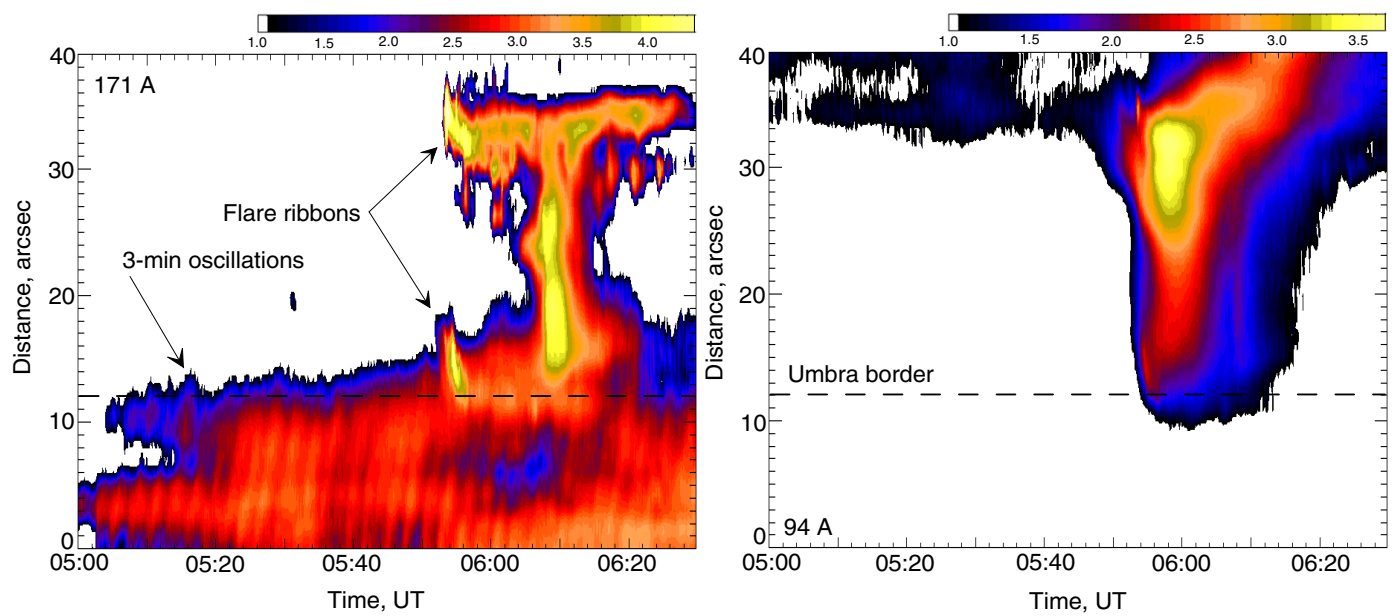

Fig. 8. Time-distance plots of flare region in $171 \AA$ (left panel) and $94 \AA$ (right panel) (SDO/AIA). In the left panel, in its bottom part, corresponding to the umbra region, there is a periodical structure of inclined wakes showing the 3-min oscillations in UV range.

at 06:10 UT (Fig. 6), and is related to the formation of the third peak on the flare profile.

Analysing the loops, we find that there is a magnetic channel that connects the sunspot with the flare energy-release site. At the beginning of the flare, the loops in the $94 \AA$ high-temperature channel were located below this channel and had a strong twist. The flare ribbons of these twisted loops were evolving quickly over the flare impulsive phase, at 05:52:30-05:55:00 UT, in association with meter and decimeter bursts (Fig. 2). The expansion of the flare loops exhibits slipping reconnection described by Aulanier et al. (2006, 2012) and Dudík et al. (2014).

Using the SDO/HMI magnetogram obtained at 06:00 UT, and the potential magnetic field extrapolation into the corona, we searched for the magnetic channel (waveguide). Although we considered only potential extrapolation, we found this magnetic channel, see Fig. 7. This magnetic channel persisted at the same location throughout the entire flare. Further, after the main phase at 06:10:36 UT, a $94 \AA$ flare loop was formed that coincided with the magnetic channel (compare the left and right parts of Fig. 7). Its new orientation shows that the twisted loops located here prior to the flare onset changed their orientation, and became oriented as the magnetic channel in potential approximation. This means that electric currents in these loops decreased during the flare, i.e. their energy was released. Note that such strong-to-weak shear transition is common during flares (e.g. Aulanier et al. 2012). Both the magnetic field lines of the channel and $94 \AA$ hot loop were anchored in the sunspot with the southern polarity, their other footpoint is connected with the northern polarity (Fig. 7).

\subsubsection{One-dimensional dynamics of waves in coronal loops}

To study the dynamics of waves propagating from the sunspot along the coronal loops into the flare region, we used timedistance plots for the $171 \AA$ and $94 \AA$ waves (Fig. 8). The left panel of Fig. 8 (171 $\AA$ ) corresponds to the coronal plasma with the temperature of $\approx 1 \mathrm{MK}$, and the right panel of Fig. 8 (94 $\AA$ ) to hot plasma with the temperature of $\approx 6 \mathrm{MK}$. The scanning direction of brightness temporal variations was selected along the wave-guiding loop starting from the sunspot, see the arrow in Fig. 7, left panel.

The left panel of Fig. 8 shows that the $\approx 3$-min waves propagate continuously from the umbral region upwards. These waves are the result of the oscillatory wakes behind propagating disturbances in the stratified atmosphere, which oscillates at the acoustic cut-off frequency (Fleck \& Schmitz 1991; Kalkofen et al. 1994). The horizontal dashed line shows the umbra boundary.

About 30 min prior to the start of the flare, an increase in the oscillatory wakes length and power occurred. This moment coincided with the increase of the oscillation power at the transition region level (Fig. 3). This indicates that the same wave process occurs both in the lower and in the upper umbra atmosphere. The wake extension led to their overrunning the umbra boundary. A new magnetic loop (Fig. 6), along which waves reach the flare region, began to be formed. The formation of the coronal loop coincided both temporally and spatially with the appearance of a new loop in the radio range (Fig. 4). An increase in the inclination of the oscillatory wakes is observed (see a series of steep features under the umbra border in Fig. 8, left panel). These changes are related to the loop heating at 06:00 UT seen well in the $94 \AA$ high-temperature channel (Fig. 8, right panel) and through an increase in the wave propagation velocity outside the umbra. We measured the inclination of the wakes along the loop near the umbra centre and at $\sim 10$ arcsec from its boundary. The calculated wave velocity was $V_{1}=36.2 \mathrm{~km} \mathrm{~s}^{-1}$ and $V_{2}=84.6 \mathrm{~km} \mathrm{~s}^{-1}$, respectively. The velocity values indicate that these waves are slow magnetosonic waves whose velocity is near the speed of sound. The kinetic temperature calculated in these two spatial regions was estimated to be $T_{1}=47700 \mathrm{~K}$ and $T_{2}=$ $260000 \mathrm{~K}$, respectively. An increase of the wave velocity (increase of the wake inclination), corresponding to a local temperature increase due to heating, is recognized as well.

\subsubsection{Spatial structure of magnetic waveguides}

To obtain a fine spatial structure of the flare loop in $171 \AA$ A, we used the PWF analysis (Sych \& Nakariakov 2008). The PWF analysis is based upon the wavelet transform described in detail by Torrence \& Compo (1998). We apply wavelet filtration to the time signal of each pixel, and decompose the signal in the time domain (time frequencies), creating a four-dimensional (two spatial coordinates, time, and frequency) data cube. Averaging over or specifying one or two of those coordinates, we determine the time-spatial distribution of the power, amplitude and phase of different spectral components to study the time evolution of the spatial-frequency information. 

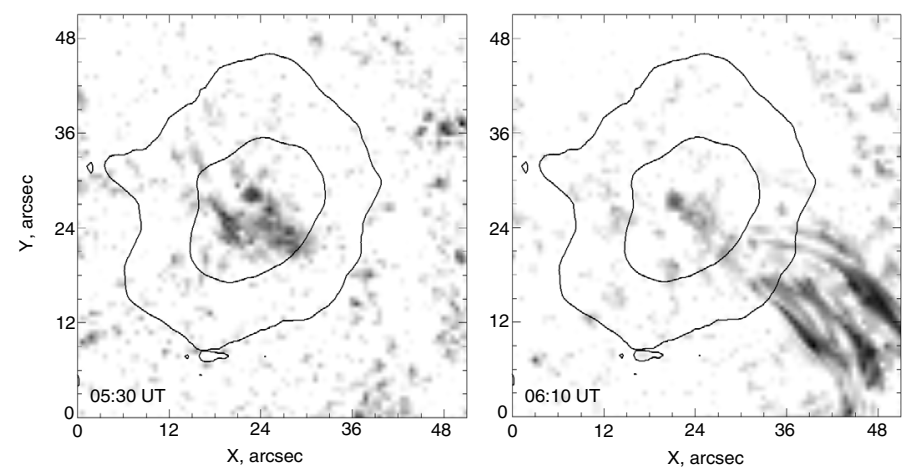

Fig. 9. Narrowband images of 3-min oscillations sources before (left panel) and maximum of the flare (right panel) made from the $171 \AA$ images.

Narrowband images of magnetic waveguides within the 1.5$3.5 \mathrm{~min}$ band were built. Prior to the flare, at 05:30 UT, the 3-min oscillatory wakes took the shape of an arrow with the footpoints in the umbra directed towards the future flare (Fig. 9). At this time, all the oscillations occurred within the umbra boundary. Following that, an extension of the wakes and their displacement into the penumbra were observed. These wakes form thin loops (Fig. 9, right) along which waves propagated into the flare region. Footpoints of these loops were anchored in the umbra. Their spatial location correlates well with the flare loop observed in the radio range (Fig. 4) and with the magnetic channel (Fig. 7).

\section{Flare scenario}

The observational data in the radio (NoRH) and ultraviolet (SDO/AIA) ranges show an explicit association between the oscillatory processes in the sunspot and the flare emergence in the adjacent region. Combining all these data with the magnetic field extrapolation, we find the magnetic channel (waveguide) connects the sunspot umbra with the flare site. The flare occurred in space under the magnetic channel. Before the flare, strongly twisted magnetic loops shows the presence of electric currents and stored free magnetic energy. Prior to the flare onset, we find a continuous increase in the power of 3-min sunspot oscillations, observed both in the transition region and in the corona. In the corona, this increase is related to the lengthening of wave propagation distance (see the wakes in Fig. 8, left panel), reaching its maximum after the flare main phase. The waves propagate from the sunspot along the magnetic channel to the flare region. Based on their period and velocities in the transition region and corona, we interpret these waves as slow $\approx 3$-min period magnetoacoustic waves. Centrifugal forces during propagation of these waves along the curved magnetic channel can lead to conversion of a portion of the longitudinal wave energy into transverse waves, a process described by Zaitsev \& Stepanov (1989). The transverse oscillations of the radio loop at the beginning of the flare support this process. In agreement with Sych et al. (2009), we propose that this process triggered the flare reconnection in space under the magnetic channel, where the magnetic field was strongly twisted. Furthermore, it seems that the waves propagating from the sunspot influence flare processes also after the flare triggering, especially during the flat stage expressed on the flare time profile in soft X-rays. These waves could also periodically influence the flare reconnection processes that lead to the formation of the flat X-ray stage. When the wave feed ended, a decrease in the X-ray radiation started. Simultaneously, the flare loop temperature decreased, as seen from the flare loop disappearance in the $94 \AA$ A high-temperature channel (6.3 MK) images, and its appearance in the cooler $171 \AA$ coronal channel (1 MK).

\section{Conclusions}

We analysed the relation between sunspot umbra oscillations and the occurrence of a small C1.5 flare in a nearby location within the active region NOAA 11494 . We used observational data at the level of the transition region (NoRH, radio) and of the corona (SDO/AIA). We performed the analysis using timedistance plots and PWF method.

The flare profiles show three peaks in the radio and in the ultraviolet radiation separated in time and altitude, as evidenced by differences in the generated emission. The flare started with type-III bursts at about $400 \mathrm{MHz}$ followed by a noise storm in the 200-300 MHz range. In soft X-rays (6-12 keV), a flat stage with an $\approx 3$-min period flux modulation was observed.

Correlation curves $(17 \mathrm{GHz})$ show sunspot radio-frequency oscillations in sunspot with the $\approx 3$-min period. The pulsations have a train character with a $12-20$-min period. Each train drifts in periods in the range of 2-4 min. The drift value decreases towards the flare onset. This indicates the formation of a spatial magnetic channel, along which slow magnetoacoustic waves start to propagate.

In the corona (SDO/AIA $171 \AA$ ), the source of the 3 -min EUV oscillations coincides with the radio loop. Its shape looks like an arrow localized in the umbra and directed towards the flare site. Further, the arrow extended, and a fine-structure rope of magnetic loops (waveguides) are formed. This rope connects the sunspot with the flare region.

The propagation length of 3-min waves starts to monotonically increase and proceeded beyond the umbra boundary $\approx 30$ min prior to the flare onset. This coincides with the oscillation peak on the $17 \mathrm{GHz}$ correlation curve and with the evolution of loops in the $171 \AA$ and $94 \AA$. The wave velocity along the loop grew from $36.2 \mathrm{~km} \mathrm{~s}^{-1}$ in the sunspot to $84.6 \mathrm{~km} \mathrm{~s}^{-1}$ in the loop leg.

The radio curves show a monotonous increase in the power of oscillations prior to the flare. We assume that this power increase is due to a local efflux of subphotospheric impulses. The power maximum was observed $30 \mathrm{~min}$ before the flare.

We propose that this enhanced flux of waves triggered the flare. However, the flare occurred not only due to this enhanced wave flux, but also because a sufficient amount of the free magnetic energy was accumulated in close vicinity of the magnetic channel prior to the flare. During the flare, the magnetic field configuration became simpler; the primary twist of loops disappeared. We assume that a formation of the flat X-ray stage is associated with an additional energy release due to quasi-periodic reconnections in the flare site, but influenced by wave processes in the magnetic channel.

Acknowledgements. This work was supported by the Russian Foundation for Basic Research under Grants 13-02-00044,13-02-90472, 14-02-91157, Marie Curie International Research Staff Exchange Scheme Fellowship within the 7th European Community Framework Programme (PIRSES-GA-2011-295272 RadioSun project), Grants P209/12/0103 and P209/12/1652 (GA CR) and the EU P-7 project No. 606862 F-CHROMA. J.D. acknowledges support from the Royal Society via the Newton International Fellowships Programme.

\section{References}

Abramov-Maximov, V. E., Gelfreikh, G. B., \& Shibasaki, K. 2011, Sol. Phys., 273,403

Alissandrakis, C. E. 1981, A\&A, 100, 197 
Aulanier, G., Pariat, E., Demoulin, P., \& DeVore, C. R. 2006, Sol. Phys., 238, 347

Aulanier, G., Janvier, M., \& Schmieder, B. 2012, A\&A, 543, A110

Bárta, M., \& Karlický, M. 2000, A\&A, 353, 757

Del Zanna, G. 2013, A\&A, 558, A73

Dudík, J., Janvier, M., Aulanier, G., et al. 2014, ApJ, 784, 144

Fleck, B., \& Schmitz, F. 1991, A\&A, 250, 1

Gary, G. A. 1989, ApJS, 69, 323

Kalkofen, W., Rossi, P., Bodo, G., et al. 1994, A\&A, 284, 3

Karlický, M., \& Jungwirth, K. 1989, Sol. Phys., 124, 319

Kotrč, P., Kupryakov, Yu. A., Kashapova, L. K., \& Bárta, M. 2013, Centr. Eur. Astrophys. Bull., 37, 555

Kupriyanova, E. G., Melnikov, V. F., \& Shibasaki, K. 2013, Sol. Phys., 284 559

Lemen, J. R., Title, A. M., Akin, D. J., et al. 2012, Sol. Phys., 257, 17

Liu, C., Lee, J., Karlický, M., et al. 2009, ApJ, 703, 757

Nakariakov, V. M., Foullon, C., Verwichte, E., \& Young, N. P. 2006, A\&A, 452, 343

Nindos, A., Kundu, M. R.,White, S. M., Shibasaki, K., \& Gopalswamy, N. 2000 ApJS, 130, 485
Norman, C. A., \& Smith, R. A. 1978, A\&A, 68, 145

Odstrčil, D., \& Karlický, M. 1997, A\&A, 326, 1252

Pesnell, W. D., Thompson, B. J., \& Chamberlin, P. C. 2012, Sol. Phys., 275, 3

Petkaki, P., Del Zanna, G., Mason, H. E., \& Bradshaw, S. J. 2012, A\&A, 547, A25

Reznikova, V. E., Shibasaki, K., Sych, R. A., \& Nakariakov, V. M. 2009, ApJ, 746, 119

Shibasaki, K. 2001, ApJ, 550, 1113

Shibasaki, K., Enome, S., Nakajima, H., et al. 1994, PASJ, 46, L17

Sych, R. A., \& Nakariakov, V. M. 2008, Sol. Phys., 248, 395

Sych, R., \& Nakariakov, V. M. 2014, A\&A, 569, A72

Sych, R., Nakariakov, V. M., Karlický, M., \& Anfinogentov, S. 2009, A\&A, 505, 791

Thompson, A. R., Moran, J. M., \& Swenson, G. W. 1986, Interferometry and Synthesis in Radio Astronomy (New York: John Wiley \& Sons) Torrence, C., \& Gilbert, P. 1998, Bull. Am. Meteorol. Soc., 79, 1

Yuan, D., Sych, R., Reznikova, V. E., \& Nakariakov, V.M. 2014, A\&A, 561, A19

Zaitsev, V. V., \& Stepanov, A. V. 1989, Sov. Astron. Lett., 15, 66

Zuccarello, F., Romano, P., Fárník, F., et al. 2009, A\&A, 493, 629 